\title{
An example of quantum imaging: rendering an object undetectable
}

\author{
Stefan Ataman ${ }^{1}$ \\ Extreme Light Infrastructure - Nuclear Physics (ELI-NP), 30 Reactorului Street, 077125 Magurele, Romania e-mail: \\ stefan. ataman@eli-np.ro
}

Received: date / Revised version: date

\begin{abstract}
In this paper we propose and analyse a Gedankenexperiment involving three non-linear crystals and two objects inserted in the idler beams. We show that, besides the behaviour that can be extrapolated from previous experiments involving two crystals and one object, we are able to predict a new effect: under certain circumstances, one of the objects can be rendered undetectable to any single detection rate on the signal photons with discarded idler photons. This effect could find applications in future developments of quantum imaging techniques.
\end{abstract}

PACS. PACS-key describing text of that key - PACS-key describing text of that key

\section{Introduction}

The non-linear optical process of spontaneous parametric down-conversion (SPDC) $[1,2,3]$ creates pairs of highly entangled single photon pairs $[4,5]$. Its use became ubiquitous in quantum optics (QO), where numerous experiments rely on this process $[6,7,8,9,10,11,12,13]$.

SPDC is a process that happens randomly, somehow in the spirit of spontaneous emission of an excited atom. However, in an experiment by $\mathrm{Ou}$, Wang, Zou and Mandel $[9,10]$, two non-linear crystals fed by the same pump laser show a phenomenon of "phase memory". A landmark experiment using two non-linear crystals was performed by Zou, Wang and Mandel [11]. Its counter-intuitive feature stemmed from the fact that the singles count rate of the two interfering signal beams shows or does not show interference effects in function of the distinguishability/indistinguishability of the discarded idler beams. Based on this principle, the imaging of small objects using undetected (discarded) photons has been recently experimentally demonstrated [12]. One can now use a wavelength where the object is transparent and detect photons of another wavelength, more convenient for the detectors [12]. This idea is pushed even further out of the lab and towards industrial applications by Kalashnikov et al. in reference [14] where they perform infrared spectroscopy yet they use red photons at the detectors. For a review on quantum imaging techniques and implementations one can consult reference [15].

With these latest developments, it becomes obvious that the field of quantum imaging is still in its infancy and many developments are to be expected in the near future.
In this paper, we show that an object can be rendered undetectable using the already proven principle of quantum imaging with discarded idler photons $[11,12]$. We propose the extension of the experiment from reference [11] to the case involving three non-linear crystals. Besides the expected behaviour (i.e. the extension of the counterintuitive features of the results of Zou et al. [11]), we show that, under certain circumstances, the second object can be rendered invisible to any photo-counters if the idler photons are discarded.

This paper is organized as follows. In Section 2 the proposed experiment is introduced and qualitatively discussed. Its complete quantum optical description is thoroughly developed in Section 3. The computation of various single and coincidence photo-detection rates is done in Section 4. Finally, conclusions are drawn in Section 5.

\section{The proposed experiment}

In the proposed Gedankenexperiment three non-linear crystals are pumped together by a common laser (see Fig. 1). We assume that the pumping power of each individual crystal can be adjusted at will. The crystals are aligned in such a way, that the idler mode of $N L_{1}\left(i_{1}\right)$ is overlapping the idler modes of $N L_{2}\left(i_{2}\right)$ and $N L_{3}\left(i_{3}\right)$. Therefore, a photo-detection at detector $D_{I}$ would not be able to tell from which nonlinear crystal the photon originated. The signal modes of $N L_{1}\left(s_{1}\right)$ and $N L_{2}\left(s_{2}\right)$ are brought together into the beam splitter $\mathrm{BS}_{a}$. Its first output leads to the photo-detector $D_{A}$, while the second one enters the beam splitter $\mathrm{BS}_{b}$, where it combines with the signal mode of $N L_{3}\left(s_{3}\right)$. The two outputs of this beam splitter lead to 


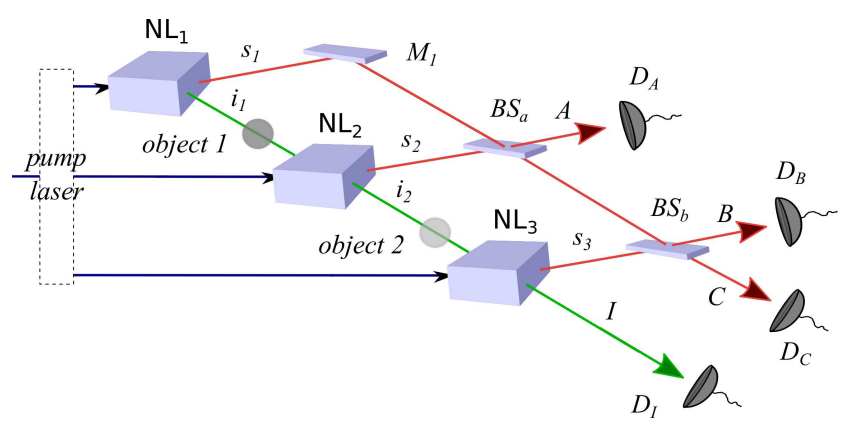

Fig. 1. The proposed experiment. Three non-linear crystals are pumped by a common laser. The idler modes of the crystals overlap, therefore become indistinguishable. The signal modes are brought to interfere through the beam splitters $\mathrm{BS}_{a}$ and $\mathrm{BS}_{b}$. The two objects can be inserted in the gray regions of $i_{1}$ and $i_{2}$.

the photo-detectors $D_{B}$ and $D_{C}$. Throughout this paper, we assume ideal and broad-band photo-detectors.

More or less transparent objects can be inserted in the dashed regions of the beams $i_{1}$ and $i_{2}$ (see Fig. 1).

If we do not pump the non-linear crystal $N L_{3}$ and consider single rates at the detector $D_{A}$, we find ourselves in the experimental setup described by Zou, Wang and Mandel [11]. Therefore, the same counter-intuitive behavior is expected, with the singles detection rate at $D_{A}$ depending on the transmissivity of the inserted object (called "object 1 ") into the beam $i_{1}$

At a first glance, the extension to three crystals and two objects seems trivial and devoid of surprises. Inserting an object (called thereafter "object 2") into the beam $i_{2}$ should be detected by a variation of the singles rate at either $D_{B}$ or $D_{C}$.

In the following, we will show that this is not always the case. For some well chosen parameters of object 1 , beam splitter $\mathrm{BS}_{a}$ and the pumping powers of $N L_{1}$ and $N L_{2}$, object 2 can simply become invisible (regardless of its transmissivity) to any single detection rate at $D_{A}, D_{B}$ or $D_{C}$. Therefore, if we discard the idler photons, there is no way to detect object 2 . Even coincidence counts $D_{B}-$ $D_{I}$ and $D_{C}-D_{I}$ fail to reveal its presence.

\section{The quantum optical description of the experiment}

In the SPDC process, the input beam (generally called "pump", $p$ ) is split into two output beams (generally called "signal" $s$ and "idler" $i$ ). This process takes place at the single photon level: one input photon is destroyed and two output photons are created. Energy $(\hbar \omega)$ and momentum $(\hbar \boldsymbol{k})$ conservation requirements (also called "phase matching conditions") impose frequency $\left(\omega_{p}=\omega_{s}+\omega_{i}\right)$ and wavenumber $\left(\boldsymbol{k}_{p} \approx \boldsymbol{k}_{s}+\boldsymbol{k}_{i}\right)$ relations between the inputoutput fields.

We shall make a number of simplifying assumptions. Similar to references $[11,12,13,20]$ we shall consider all modes as being monochromatic. Since the phase relation between the three pump fields can be fixed at any value, we simply assume that all crystals are pumped in phase, yielding

$$
\begin{array}{r}
\left|\psi_{\text {in }}\right\rangle=\frac{1}{\mathcal{N}}\left(\gamma_{1}\left|1_{p_{1}}\right\rangle+\gamma_{2}\left|1_{p_{2}}\right\rangle+\gamma_{3}\left|1_{p_{3}}\right\rangle\right) \\
=\frac{\left(\gamma_{1} \hat{a}_{p_{1}}^{\dagger}+\gamma_{2} \hat{a}_{p_{2}}^{\dagger}+\gamma_{3} \hat{a}_{p_{3}}^{\dagger}\right)}{\mathcal{N}}|0\rangle
\end{array}
$$

where $\mathcal{N}$ is a normalization constant, $\left|1_{p_{k}}\right\rangle$ denotes a Fock state with one photon in mode (port) $p_{k}, \hat{a}_{p_{k}}^{\dagger}$ denotes the usual input creation operator in mode $p_{k}$ with $k=1,2,3$ and $|0\rangle$ is the vacuum state. A detailed discussion of this choice is given in Appendix A. The $\gamma_{k}$ parameters with $k=1,2,3$ (assumed real and positive throughout this paper) are connected to input power differences for identical non-linear crystals $[21,22]$. The quantum state (1) also implies that no two down-conversions happen at the same moment. This is consistent with the low down-conversion efficiency $[9,11,13]$ of the non-linear crystals.

From the perspective of QO, the field operator transformation in each SPDC process is

$$
\hat{a}_{p_{n}}^{\dagger} \rightarrow \hat{a}_{s_{n}}^{\dagger} \hat{a}_{i_{n}}^{\dagger}
$$

where $\hat{a}_{s_{n}}^{\dagger}\left(\hat{a}_{i_{n}}^{\dagger}\right)$ denotes the signal (idler) creation operators and $n=1,2,3$. The process of SPDC creates pairs of photons from a pump photon in a spontaneous, hence unpredictable way. However, in our model (1) a fixed phase relationship is assumed among the parametric down-conversions (and set to zero for convenience).

With the two objects inserted, the experiment is depicted in Fig. 2. Each object is modelled through a beam splitter [16] having transmission (reflection) coefficients $T_{m}\left(R_{m}\right)$ and a phase shift $\varphi_{m}$ with $m=1,2$. Typical BS unitarity constrains [17] apply to these coefficients. The inputs $u_{m}$ are always in the vacuum mode while the fictitious modes $v_{m}$ have to be considered, for reasons of unitary evolution of the system (although in the final computation they will be traced out). For a perfectly transparent (opaque) object $m$ we have $T_{m}=1\left(T_{m}=0\right)$ with $m=1,2$.

For simplicity, in the main part of the paper, the optical lengths of $s_{1}$ and $s_{2}$ leading to $\mathrm{BS}_{a}$ are assumed to be equal. The same assumption is taken for $s_{2}$ and $s_{3}$. The scenario with unequal lengths in the signal beams is discussed in Appendix B.

Beam splitters $\mathrm{BS}_{a}$ and $\mathrm{BS}_{b}$ are characterized by the transmission (reflection) coefficients $T_{n}\left(R_{n}\right)$ with $n=$ $\{a, b\}$. Unitarity requirements impose the well-known conditions $\left|T_{n}\right|^{2}+\left|R_{n}\right|^{2}=1$ and $T_{n} R_{n}^{*}+T_{n}^{*} R_{n}=0$ [17]. Whenever we shall assume balanced beam splitters, we shall replace $T_{n}=1 / \sqrt{2}$ and $R_{n}=i / \sqrt{2}$.

After a series of calculations (see details for a slightly more general case in Appendix B), the output state vector 


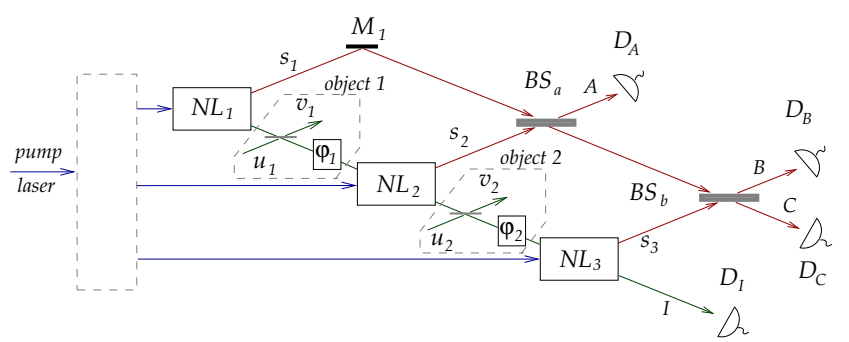

Fig. 2. The objects inserted in the idler beams of $N L_{1}$ and, respectively, $N L_{2}$ can be modelled by two beam splitters having transmissivity (reflectivity) coefficient $T_{k}\left(R_{k}\right)$ and two phase delays $\varphi_{k}$ with $k=1,2$.

is found to be

$$
\begin{array}{r}
\left|\psi_{\text {out }}\right\rangle=\frac{1}{\mathcal{N}}\left(T_{2} \mathrm{e}^{i \varphi_{2}}\left(\gamma_{1} R_{a} T_{1} \mathrm{e}^{i \varphi_{1}}+\gamma_{2} T_{a}\right)\left|1_{A} 1_{I}\right\rangle\right. \\
+R_{2}\left(\gamma_{1} R_{a} T_{1} \mathrm{e}^{i \varphi_{1}}+\gamma_{2} T_{a}\right)\left|1_{A} 1_{v_{2}}\right\rangle \\
+\gamma_{1} R_{a} R_{1}\left|1_{A} 1_{v_{1}}\right\rangle \\
+\left(R_{b} T_{2} \mathrm{e}^{i \varphi_{2}}\left(\gamma_{1} T_{a} T_{1} \mathrm{e}^{i \varphi_{1}}+\gamma_{2} R_{a}\right)+\gamma_{3} T_{b}\right)\left|1_{B} 1_{I}\right\rangle \\
+R_{b} R_{2}\left(\gamma_{1} T_{a} T_{1} \mathrm{e}^{i \varphi_{1}}+\gamma_{2} R_{a}\right)\left|1_{B} 1_{v_{2}}\right\rangle \\
+\gamma_{1} T_{a} R_{b} R_{1}\left|1_{B} 1_{v_{1}}\right\rangle \\
+\left(T_{b} T_{2} \mathrm{e}^{i \varphi_{2}}\left(\gamma_{1} T_{a} T_{1} \mathrm{e}^{i \varphi_{1}}+\gamma_{2} R_{a}\right)+\gamma_{3} R_{b}\right)\left|1_{C} 1_{I}\right\rangle \\
+T_{b} R_{2}\left(\gamma_{1} T_{a} T_{1} \mathrm{e}^{i \varphi_{1}}+\gamma_{2} R_{a}\right)\left|1_{C} 1_{v_{2}}\right\rangle \\
\left.+\gamma_{1} T_{a} T_{b} R_{1}\left|1_{C} 1_{v_{1}}\right\rangle\right)
\end{array}
$$

where $\left|1_{A} 1_{I}\right\rangle$ denotes a Fock state with one photon in mode (port) $A$ and one in mode $I$ etc. We construct the density matrix $\hat{\rho}_{\text {out }}=\left|\psi_{\text {out }}\right\rangle\left\langle\psi_{\text {out }}\right|$ and trace it out over the fictitious modes $v_{1}$ and $v_{2}$, yielding the reduced density matrix

$$
\hat{\rho}_{\text {red }}=\operatorname{Tr}_{v_{1}, v_{2}}\left\{\hat{\rho}_{\text {out }}\right\}=\sum_{m, n}\left\langle m_{v_{1}} n_{v_{2}}\left|\hat{\rho}_{\text {out }}\right| m_{v_{1}} n_{v_{2}}\right\rangle
$$

\section{Singles and coincidence rates evaluation}

Having the reduced density matrix (4) allows one to make all predictions regarding the probabilities of singles or coincidence detections. For example, the singles detection probability at the photo-detector $D_{A}$ is given by

$$
P_{A}=\operatorname{Tr}\left\{\hat{a}_{A}^{\dagger} \hat{a}_{A} \hat{\rho}_{\text {red }}\right\}
$$

and after a short computation one arrives at

$$
P_{A} \sim\left|\gamma_{1} R_{a} T_{1} \mathrm{e}^{i \varphi_{1}}+\gamma_{2} T_{a}\right|^{2}+\gamma_{1}^{2}\left|R_{a} R_{1}\right|^{2}
$$

It is noteworthy to mention that the dependence on $T_{2}$ $\left(R_{2}\right)$ disappears during this computation, therefore the final result from equation (6), as expected, does not depend on the properties of object 2 . If one assumes equal pumping powers to $N L_{1}$ and $N L_{2}$ (i.e. $\gamma_{1}=\gamma_{2}$ ) and a balanced beam splitter $\mathrm{BS}_{a}$, equation (6) simplifies to

$$
P_{A} \sim 1-T_{1} \sin \left(\varphi_{1}\right)
$$

where we assumed $T_{1}$ to be real. This result is similar to the ones already reported $[11,12]$. Its paradoxical nature arises from the fact that the two idler modes $\left(i_{1}\right.$ and $\left.i_{2}\right)$ do not participate in the interference of the signal modes $\left(s_{1}\right.$ and $\left.s_{2}\right)$. However, their indistinguishability/distinguishability allows one to have or not to have a quantum state that can be factorized. It is worthwhile to note that the distinguishability/indistinguishability of the beams $i_{2}$ and $i_{3}$ at the detector $D_{I}$ plays no role in measuring the probability of detection at $P_{A}$.

The singles detection probability at $D_{B}$ is given by $P_{B}=\operatorname{Tr}\left\{\hat{a}_{B}^{\dagger} \hat{a}_{B} \hat{\rho}_{\text {red }}\right\}$ and one obtains

$$
\begin{gathered}
P_{B} \sim\left|R_{b} T_{2} \mathrm{e}^{i \varphi_{2}}\left(\gamma_{1} T_{a} T_{1} \mathrm{e}^{i \varphi_{1}}+\gamma_{2} R_{a}\right)+\gamma_{3} T_{b}\right|^{2} \\
+\left|R_{b} R_{2}\left(\gamma_{1} T_{a} T_{1} \mathrm{e}^{i \varphi_{1}}+\gamma_{2} R_{a}\right)\right|^{2}+\gamma_{1}^{2}\left|T_{a} R_{b} R_{1}\right|^{2}
\end{gathered}
$$

At first sight, this result is simply the extension of equation (6) to the case involving three crystals and two objects. However, one can select values of $\gamma_{1}, \gamma_{2}, T_{a}, T_{1}$ and $\varphi_{1}$ so that

$$
\gamma_{1} T_{a} T_{1} \mathrm{e}^{i \varphi_{1}}+\gamma_{2} R_{a}=0
$$

In this case, equation (8) simplifies to

$$
P_{B} \sim \gamma_{1}^{2}\left|T_{a} R_{b} R_{1}\right|^{2}+\gamma_{3}^{2}\left|T_{b}\right|^{2}
$$

In other words, the presence or absence of object 2 has no influence on the singles photo-count result at $D_{B}$. A similar result is obtained at the detector $D_{C}$, namely

$$
P_{C} \sim \gamma_{1}^{2}\left|T_{a} T_{b} R_{1}\right|^{2}+\gamma_{3}^{2}\left|R_{b}\right|^{2}
$$

Even more counter-intuitive is the coincidence count result at detectors $D_{B}$ and $D_{I}$, yielding

$$
P_{B-I} \sim \gamma_{3}^{2}\left|T_{b}\right|^{2}
$$

or, similarly, at detectors $D_{C}$ and $D_{I}$, giving

$$
P_{C-I} \sim \gamma_{3}^{2}\left|R_{b}\right|^{2}
$$

The question arises: did object 2 become completely undetectable if we obey the condition from equation (9)? The answer is "almost". With the current configuration, we could probe the presence of object 2 by measuring the single counts at $D_{I}$ or the coincidence counts at $D_{A}$ and $D_{I}$. The latter, for example, yields

$$
P_{A-I} \sim \gamma_{1}^{2}\left|T_{1} T_{2}\right|^{2} /\left|R_{a}\right|^{2}
$$

a result depending, indeed, on $T_{2}$. However, if we discard the idler photons (as done in reference [12]), object 2 is completely invisible to any single detection rates. 


\section{Conclusions}

In this paper we proposed and discussed an experiment able to render undetectable - using quantum, rather than classical principles - a more or less transparent object. For well chosen experimental parameters (including only the first object), a condition for the undetectability of the second object can be obtained. In this case, no matter what properties the second object has, its presence or absence will remain undetected to all single detection rates if the idler photons are discarded.

\section{Acknowledgments}

The author wishes to thank Dr. Radu Ionicioiu for useful suggestions for the final form of the manuscript and Dr. Waleed Mouhali for helping him double-check the main results of this paper. The author also wishes to thank the anonymous reviewers for their help in improving this paper.

\section{A About the input state of the system}

Equation (1) might seem to be awkward, since the inputs of the non-linear crystals are actually intense coherent fields, while we consider them as being single-photon Fock states. In order to justify this choice, we first write the input state as a coherent state $\left|\alpha_{p_{n}}\right\rangle$ with $n=1,2,3$ and expanding it in Fock state yields

$\left|\alpha_{p_{n}}\right\rangle=e^{-\left|\alpha_{p_{n}}\right|^{2} / 2}\left(|0\rangle+\alpha_{p_{n}} \hat{a}_{p_{n}}^{\dagger}|0\rangle+\frac{\left(\alpha_{p_{n}} \hat{a}_{p_{n}}^{\dagger}\right)^{2}}{2}|0\rangle+\ldots\right)$

The only term from equation (15) that yields two photons after the the down-conversion process (2) is the second one. This down-conversion process has a (typically low) efficiency $g_{n}$ where this coefficient is connected to the $\chi^{(2)}$ nonlinearity of each crystal. Therefore, if we denote $\gamma_{n}=$ $\left|\alpha_{n}\right| g_{n}$ and assume post-selection of the two-photon state only (higher order nonlinear processes are negligible) takes us to the input state given by equation (1). If one wants to avoid any higher-order nonlinear process in the crystals, number-resolving photo-detectors (e. g. superconducting nanowire [23]) can be used instead of the usual ones.

A similar result for the output state vector can be obtained by considering the signal and idler modes initially in the vacuum mode and by applying a parametric interaction Hamiltionian $[11,15]$.

\section{B Computing the field operator transformations}

Contrary to Section 3, we assume the lengths of the signal beams to the beam splitters non-equal. Therefore, for example, the path length difference (delay) in the beams

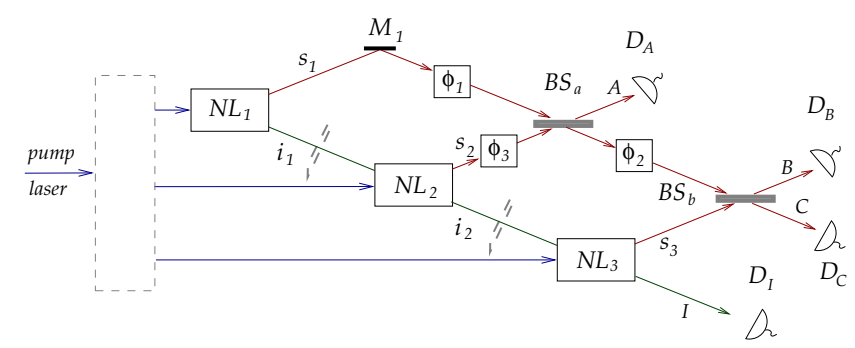

Fig. 3. The experiment in the general case. Path length differences are modelled through phase delays $\phi_{1}, \phi_{2}$ and $\phi_{3}$, as depicted above.

$s_{1}$ and $s_{2}$ is modelled by the phase shift $\phi_{1}$. Additional delays can be added, as depicted in Fig. 3 .

The singles/coincidence detection rates we are interested in can be obtained in several ways. One could follow the approach from reference [13] or the one from reference [20]. Here, we sketch another way of computing them, using a graphical method introduced in [18] and extended to non-linear optics in [19]. We can draw the graph associated to our experiment as depicted in Fig. 4 and one obtains the field operator transformations

$$
\begin{gathered}
\hat{a}_{p_{1}}^{\dagger}=\gamma_{1}\left(R_{a} \mathrm{e}^{i \phi_{1}} \hat{a}_{A}^{\dagger}+T_{a} R_{b} \mathrm{e}^{i\left(\phi_{1}+\phi_{2}\right)} \hat{a}_{B}^{\dagger}+T_{a} T_{b} \mathrm{e}^{i\left(\phi_{1}+\phi_{2}\right)} \hat{a}_{C}^{\dagger}\right) \\
\times\left(T_{1} \mathrm{e}^{i \varphi_{1}} T_{2} \mathrm{e}^{i \varphi_{2}} \hat{a}_{I}^{\dagger}+T_{1} \mathrm{e}^{i \varphi_{1}} \hat{a}_{v_{2}}^{\dagger}+R_{1} \hat{a}_{v_{1}}^{\dagger}\right)(16) \\
\hat{a}_{p_{2}}^{\dagger}=\gamma_{2}\left(T_{a} \mathrm{e}^{i \phi_{3}} \hat{a}_{A}^{\dagger}+R_{a} R_{b} \mathrm{e}^{i\left(\phi_{2}+\phi_{3}\right)} \hat{a}_{B}^{\dagger}+R_{a} T_{b} \mathrm{e}^{i\left(\phi_{2}+\phi_{3}\right)} \hat{a}_{C}^{\dagger}\right) \\
\times\left(T_{2} \mathrm{e}^{i \varphi_{2}} \hat{a}_{I}^{\dagger}+R_{2} \hat{a}_{v_{2}}^{\dagger}\right)(17) \\
\hat{a}_{p_{3}}^{\dagger}=\gamma_{3}\left(T_{b} \hat{a}_{B}^{\dagger}+R_{b} \hat{a}_{C}^{\dagger}\right) \hat{a}_{I}^{\dagger}
\end{gathered}
$$

Starting from the input state vector (1) and applying the field operator transformations (16)-(18), after some straightforward algebra one gets the output state vector

$$
\begin{array}{r}
\left|\psi_{\text {out }}\right\rangle=\frac{1}{\mathcal{N}}\left(T_{2} \mathrm{e}^{i \varphi_{2}}\left(\gamma_{1} R_{a} T_{1} \mathrm{e}^{i \phi_{1}} \mathrm{e}^{i \varphi_{1}}+\gamma_{2} T_{a} \mathrm{e}^{i \phi_{3}}\right)\left|1_{A} 1_{I}\right\rangle\right. \\
+\left(\gamma_{1} R_{a} T_{1} R_{2} \mathrm{e}^{i \varphi_{1}} \mathrm{e}^{i \phi_{1}}+\gamma_{2} T_{a} R_{2} \mathrm{e}^{i \phi_{3}}\right)\left|1_{A} 1_{v_{2}}\right\rangle \\
+\gamma_{1} R_{a} R_{1} \mathrm{e}^{i \phi_{1}}\left|1_{A} 1_{v_{1}}\right\rangle \\
+\left(R_{b} T_{2} \mathrm{e}^{i \phi_{2}} \mathrm{e}^{i \varphi_{2}}\left(\gamma_{1} T T_{1} \mathrm{e}^{i \phi_{1}} \mathrm{e}^{i \varphi_{1}}+\gamma_{2} R_{a} \mathrm{e}^{i \phi_{3}}\right)+\gamma_{3} T_{b}\right)\left|1_{B} 1_{I}\right\rangle \\
+R_{b} R_{2} \mathrm{e}^{i \phi_{2}}\left(\gamma_{1} T_{a} T_{1} \mathrm{e}^{i \phi_{1}} \mathrm{e}^{i \varphi_{1}}+\gamma_{2} R_{a} \mathrm{e}^{i \phi_{3}}\right)\left|1_{B} 1_{v_{2}}\right\rangle \\
+\gamma_{1} T_{a} R_{b} R_{1} \mathrm{e}^{i \phi_{1}} \mathrm{e}^{i \phi_{2}}\left|1_{B} 1_{v_{1}}\right\rangle \\
+\left(T_{b} T_{2} \mathrm{e}^{i \phi_{2}} \mathrm{e}^{i \varphi_{2}}\left(\gamma_{1} T_{a} T_{1} \mathrm{e}^{i \phi_{1}} \mathrm{e}^{i \varphi_{1}}+\gamma_{2} R_{a} \mathrm{e}^{i \phi_{3}}\right)+\gamma_{3} R_{b}\right)\left|1_{C} 1_{I}\right\rangle \\
+T_{b} R_{2} \mathrm{e}^{i \phi_{2}}\left(\gamma_{1} T_{a} T_{1} \mathrm{e}^{i \phi_{1}} \mathrm{e}^{i \varphi_{1}}+\gamma_{2} R_{a} \mathrm{e}^{i \phi_{1}}\right)\left|1_{C} 1_{v_{2}}\right\rangle \\
\left.+\gamma_{1} T_{a} T_{b} R_{1} \mathrm{e}^{i\left(\phi_{1}+\phi_{2}\right)}\left|1_{C} 1_{v_{1}}\right\rangle\right)
\end{array}
$$

where $\mathcal{N}$ is a normalization constant. By imposing $\phi_{1}=$ $\phi_{2}=\phi_{3}=0$ equation (19) migrates into equation (3) from Section 3. 


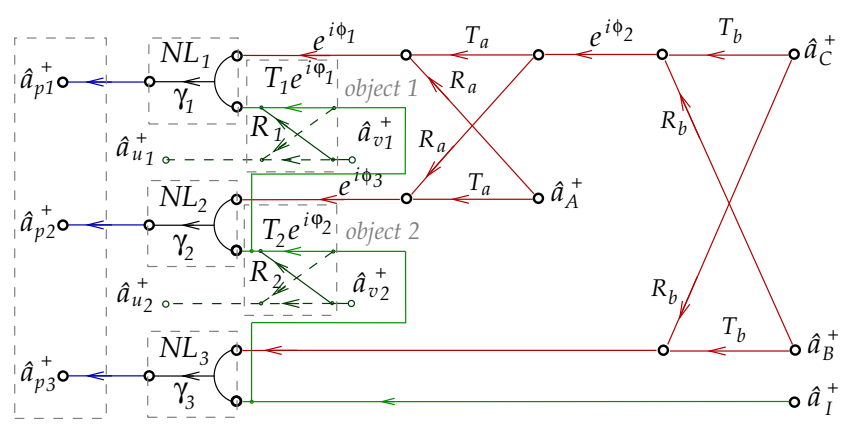

Fig. 4. The proposed Gedankenexperiment described with the graphical method. We are interested in finding the input-output (creation) field operator transformations $\hat{a}_{p_{k}}^{\dagger}=$ $f_{k}\left(\hat{a}_{A}^{\dagger}, \hat{a}_{B}^{\dagger}, \hat{a}_{C}^{\dagger}, \hat{a}_{I}^{\dagger}\right)$ i.e. the (operator) functions $f_{k}$ with $k=$ $1,2,3$.

\section{Generalization of the previous results}

We generalize here the results from Section 4. Using the output wavevector from equation (19) and following the same approach from Section 4 , the singles detection probability at detector $D_{B}$ is found to be

$$
P_{B} \sim\left|R_{b} T_{2} \mathrm{e}^{i \phi_{2}} \mathrm{e}^{i \varphi_{2}}\left(\gamma_{1} T_{a} T_{1} \mathrm{e}^{i \phi_{1}} \mathrm{e}^{i \varphi_{1}}+\gamma_{2} R_{a} \mathrm{e}^{i \phi_{3}}\right)+\gamma_{3} T_{b}\right|^{2}
$$$$
+\left|R_{b} R_{2}\right|^{2}\left|\gamma_{1} T_{a} T_{1} \mathrm{e}^{i \phi_{1}} \mathrm{e}^{i \varphi_{1}}+\gamma_{2} R_{a} \mathrm{e}^{i \phi_{3}}\right|^{2}+\gamma_{1}^{2}\left|T_{a} R_{b} R_{1}\right|^{2}(20)
$$

5. P. Kwiat, K. Mattle, H. Weinfurter, A. Zeilinger, A. Sergienko, Y. Shih, Phys. Rev. Lett. 75, 4337 (1995)

6. C. K. Hong, Z. Y. Ou, L. Mandel, Phys. Rev. Lett., 59, 2044 (1987)

7. T. Pittman et al., Phys. Rev. Let. 77, 10 (1996)

8. Z. Y. Ou, X. Y. Zou, L. J. Wang, L. Mandel, Phys. Rev. Lett., 65, 321 (1990)

9. Z. Y. Ou, L. J. Wang, L. Mandel, Phys. Rev. A, 40, 1428 (1989)

10. Z. Y. Ou, L. J. Wang, X. Y. Zou, L. Mandel, Phys. Rev. A, 41, 566 (1990)

11. X. Y. Zou, L. J. Wang, L. Mandel, Phys. Rev. Lett. 67, 318 (1991)

12. G. B. Lemos et al., Nature, 512, 409 (2014)

13. A. Heuer, R. Menzel, P. W. Milonni, Phys. Rev. Lett. 114, $053601(2015)$

14. D. A. Kalashnikov et al., Nature Photonics, 10, 98 (2016)

15. D. S. Simon et al., Int. J. Quantum Inf., 12, 1430004 (2014)

16. U. Leonhardt, Rep. Prog. Phys. 66, 1207 (2003)

17. R. Loudon, The Quantum Theory of Light, (Oxford University Press, Third Edition, 2003)

18. S. Ataman, Eur. Phys. J. D 68, 288 (2014)

19. S. Ataman, Eur. Phys. J. D 69, 44 (2015)

20. H. M. Wiseman, K. Mølmer, Phys. Lett. A 270, 245 (2000)

21. T. J. Herzog, J. G. Rarity, H. Weinfurter, A. Zeilinger, Phys. Rev. Lett. 72, 629 (1994)

22. Ö. Süzer, T.G. Goodson III, Opt. Express. 16, 20166 (2008)

23. A. Divochiy et al., Nature Photonics 2, 302 (2008)

The condition given by equation (9) for making object 2 invisible becomes in this case

$$
\gamma_{1} T_{a} T_{1} \mathrm{e}^{i \phi_{1}} \mathrm{e}^{i \varphi_{1}}+\gamma_{2} R_{a} \mathrm{e}^{i \phi_{3}}=0
$$

It is obvious now that the delays bring no new elements in our condition: the phase shifts $\phi_{1}$ and $\phi_{3}$ can be grouped together and eventually absorbed into $\varphi_{1}$. Moreover, $\phi_{2}$ is useless in respect with any invisibility condition regarding object 2. If condition (21) is satisfied, the single photocount probability at $D_{B}$ is

$$
P_{B} \sim \gamma_{1}^{2}\left|T_{a} R_{b} R_{1}\right|^{2}+\gamma_{3}^{2}\left|T_{b}\right|^{2}
$$

The coincidence counts at the detectors $D_{B}$ and $D_{I}$ can be equally computed yielding $P_{B-I} \sim \gamma_{3}^{2}\left|T_{b}\right|^{2}$. It is not difficult to show that the condition for cancelling the dependence of the coincidence counts at $D_{A}$ and $D_{I}$ on $T_{2}$ (i.e. $\gamma_{1} R_{a} T_{1} \mathrm{e}^{i \phi_{1}} \mathrm{e}^{i \varphi_{1}}+\gamma_{2} T_{a} \mathrm{e}^{i \phi_{3}}=0$ ) cannot be simultaneously satisfied with equation (21).

\section{References}

1. D. C. Burnham, D. L. Weinberg, Phys. Rev. Lett. 25, 84 (1970)

2. D. N. Klyshko, Sov. Phys. JETP Lett. 6, 23 (1967)

3. C. K. Hong, L. Mandel, Phys. Rev. A, 31, 2409 (1985)

4. M. H. Rubin, D. N. Klyshko, Y. H. Shih, A. V. Sergienko, Phys. Rev. A, 50, 5122 (1994); W. P. Grice, I. A. Walmsley, Phys. Rev. A, 56, 1627 (1997); T. E. Keller, M. H. Rubin, Phys. Rev. A, 56, 1534 (1997) 